Claremont Colleges

Scholarship@ Claremont

All HMC Faculty Publications and Research

HMC Faculty Scholarship

$11-1-2010$

\title{
Engineering Flow States with Localized Forcing in a Thin, Marangoni-Driven Inclined Film
}

Rachel Levy

Harvey Mudd College

Stephen Rosenthal '09

Harvey Mudd College

Jeffrey Wong '11

Harvey Mudd College

\section{Recommended Citation}

Levy, R, Rosenthal, S, Wong, J. Engineering flow states with localized forcing in a thin Marangoni-driven inclined film. Phys Rev E. 2010;82(5): 1-12.

This Article is brought to you for free and open access by the HMC Faculty Scholarship at Scholarship @ Claremont. It has been accepted for inclusion in All HMC Faculty Publications and Research by an authorized administrator of Scholarship @ Claremont. For more information, please contact scholarship@cuc.claremont.edu. 


\title{
Engineering flow states with localized forcing in a thin Marangoni-driven inclined film
}

\author{
Rachel Levy, ${ }^{*}$ Stephen Rosenthal, ${ }^{\dagger}$ and Jeffrey Wong ${ }^{*}$ \\ Department of Mathematics, Harvey Mudd College, 301 Platt Blvd., Claremont, CA, 91711 USA
}

(Received 17 August 2010; published 16 November 2010)

\begin{abstract}
Numerical simulations of lubrication models provide clues for experimentalists about the development of wave structures in thin liquid films. We analyze numerical simulations of a lubrication model for an inclined thin liquid film modified by Marangoni forces due to a thermal gradient and additional localized forcing heating the substrate. Numerical results can be explained through connections to theory for hyperbolic conservation laws predicting wave fronts from Marangoni-driven thin films without forcing. We demonstrate how a variety of forcing profiles, such as Gaussian, rectangular, and triangular, affect the formation of downstream transient structures, including an $N$ wave not commonly discussed in the context of thin films. Simulations employing a controlled approximation of a compressive-undercompressive wave pair demonstrate possibilities for applications of localized forcing as microfluidic valve. In the simulations, localized forcing provides a control parameter that can be used to determine mass flux and film profiles.
\end{abstract}

DOI: 10.1103/PhysRevE.82.056320

PACS number(s): 47.15.gm, 68.15.+e, 47.85.mb

\section{INTRODUCTION}

Thin liquid films driven up an inclined plane by temperature-induced surface-tension gradients have been the focus of extensive mathematical and experimental research. Using the lubrication approximation, the motion of the film is described by a single fourth-order partial differential equation (PDE) that models the evolution of the height of the film in one spatial dimension and time. Such films are of particular interest because they are known to exhibit both classical and nonclassical waves. Bertozzi et al. first proved the existence of nonclassical undercompressive waves in such films [1]. Experimental evidence of these waves, including a lack of fingering instability at the leading edge of the film and a widening capillary ridge, was provided by Cazabat et al. [2] and later by Sur et al. [3]. A theory developed by LeFloch and Shearer [4] for the mechanism generating undercompressive waves was applied by Levy and Shearer [5] to classify the types of wave structures emerging from a Marangonidriven film without additional forcing.

In this work, we connect the theory of Shearer and LeFloch to a model proposed by Haskett et al. [6] for a locally driven thin film. Beginning with a thin flow of Marangoni (surface tension) and gravity-driven viscous fluid, a localized forcing is applied, which could be implemented in a physical experiment using a laser sheet [7]. The localized forcing is envisioned for microfluidic coating flows as a way to manipulate the liquid coating without direct contact by another solid. Other related applications include cleaning of a surface using a laser and a thin liquid film [8] and Marangoni drying $[9,10]$.

This work employs numerical simulations to explore open questions in [6] about how localized forcing acts as a "microfluidic valve." An examination of transient solutions in numerical simulations reveals classical structures, including

\footnotetext{
*levy@hmc.edu

†srosenthal@hmc.edu

†jtwong@hmc.edu
}

an $N$ wave, that appear as the steady-state solution is forming. $N$ waves are familiar solutions of hyperbolic PDEs, such as the inviscid Burgers equation and are known in applications such as gas dynamics [11], but have not been discussed in the context of thin liquid films. We also explore a bifurcation between two types of solutions corresponding to weak and strong forcing and the effect of the forcing on an incoming mass of fluid. For both types of solutions, we connect the inner solution proposed in [6] to an outer solution that is fully described by the choice of boundary conditions and the theory developed in [5] for the films without localized forcing.

In Sec. I A, we review the equation modeling a thin film without localized forcing. In Sec. I B, we review the relevant results from theory developed for hyperbolic conservation laws modeling thin liquid films. In Sec. I C, we model the addition of localized forcing. In Sec. II A, we discuss steadystate solutions from constant initial conditions. In Sec. II B, we analyze transient $N$ waves from constant initial conditions and simulations from several forcing profiles. In Sec. III, we introduce a controlled nonmonotonic initial condition and analyze transient and steady-state solutions that evolve in numerical simulations. We conclude with a discussion of how localized forcing serves as a control parameter in numerical simulations and has potential application as a control mechanism for coating flows.

\section{A. Thin-film experiment without localized forcing}

This research is motivated by physical experiments of thin liquid films driven by Marangoni stresses. The experiments first focused on a thin film driven up an inclined plane and later on the same type of film with localized forcing modifying the flow. We first describe the experiment, modeling, and theory for flow without localized forcing to provide a context for numerical simulations with forcing.

Figure 1 contains a schematic of the physical experiment described in [3], in which a thin film is driven up an inclined substrate by a surface stress, induced by a surface-tension gradient, known as a Marangoni force. The substrate is a 


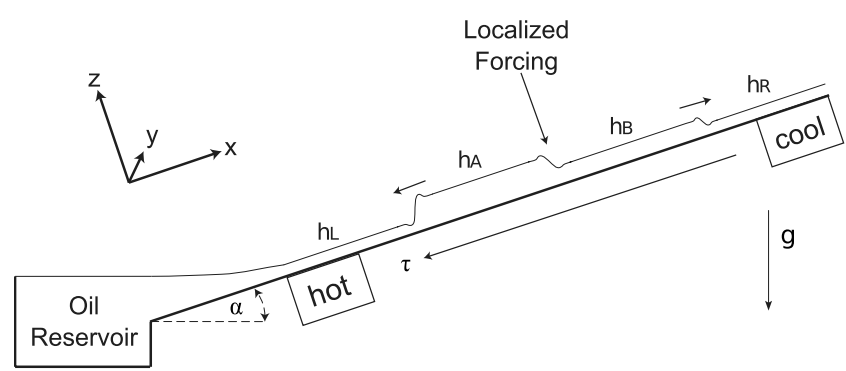

FIG. 1. Diagram of the physical thin-film experiment from [6]. Liquid advances from the reservoir up the inclined plane due to temperature gradient $\tau$.

silicon wafer mounted on a brass plate, inclined at an angle $\alpha$ from the horizontal. The constant $g$ is acceleration due to gravity. The silicon wafer is prewetted with a layer of polydimethylsiloxane silicone oil and wiped, so that only a small residual layer remains. To create a Marangoni force, the substrate is heated at the bottom end of the plate and cooled at the top end until a constant temperature gradient $\tau$ (linear temperature profile) is established. At the top end of the wafer where the temperature is lower, the surface tension of the fluid is higher. By imposing a large enough temperature gradient, the Marangoni surface stress drives the fluid slowly up the plate. The flow is quite viscous and slow: with a temperature gradient of $20{ }^{\circ} \mathrm{C} / \mathrm{cm}$, the film climbs at a rate of 2-20 $\mathrm{mm} / \mathrm{h}$, depending on the inclination angle.

We consider a one-dimensional model for the evolution of a film of height $h(x, t)$ that emerges from a reservoir at the bottom of the substrate. By prewetting the substrate and imposing the condition $h(x, t)>0$, stress singularities at the contact line are avoided [12]. The flow without localized forcing is modeled by a nondimensionalized scalar PDE for the evolution of the film height $h(x, t)$ as it evolves in space $x$ and time $t$. The nondimensionalized thin-film equation,

$$
\frac{\partial h}{\partial t}+\frac{\partial}{\partial x}\left(h^{2}-h^{3}\right)=-\frac{\partial}{\partial x}\left(h^{3} \frac{\partial^{3} h}{\partial x^{3}}\right),
$$

is derived in [6] using the well-known lubrication approximation [13] and a depth-averaged velocity.

The nondimensionalization is standard in [6]: the dimensioned quantities $\hat{x}, \hat{h}$, and $\hat{t}$ are scaled as $\hat{x}=L x, \hat{h}=H h$, and $\hat{t}=L / U t$ where $L, H$, and $U$ are the characteristic length, height, and tangential velocity. These are assigned the values

$$
L=\left(\frac{3 \hat{\sigma}_{0} \gamma \tau}{2 \rho^{2} g^{2} \sin ^{2} \alpha}\right)^{1 / 3}, \quad H=\frac{3 \gamma \tau}{2 \rho g \sin \alpha}, \quad U=\frac{3 \gamma^{2} \tau^{2}}{4 \mu \rho g \sin \alpha},
$$

where $\gamma$ is the variation in surface tension relative to temperature (assumed constant in the model), $\tau$ is the temperature gradient strength, $\rho$ is the fluid density, $g$ is the acceleration due to gravity, $\alpha$ is the inclination angle, $\hat{\sigma}_{0}$ is the surface tension of the fluid at ambient temperature, and $\mu$ is the dynamic fluid viscosity. In deriving the model the other relevant scalings are for vertical velocity $w$ and pressure $p$, given by $\hat{w}=(H U / L) w$ and $\hat{p}=\left(\mu U L / H^{2}\right) p$.

As shown in Fig. 1, $x$ is oriented up the substrate, in the direction of the Marangoni force. The left-hand terms are a

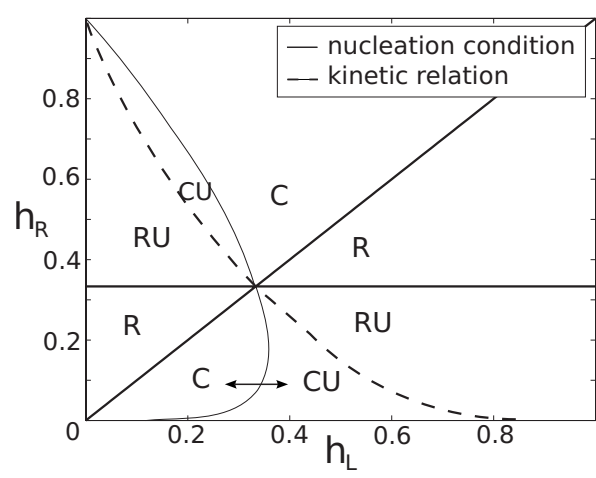

FIG. 2. The Riemann map predicts the types of wave structures that develop from Eq. (1), boundary conditions $\left(h_{L}, h_{R}\right)$, and a monotonic initial profile. Here, $\mathrm{C}$ stands for a compressive wave, $\mathrm{U}$ for an undercompressive wave, and $\mathrm{R}$ for a rarefaction. $\mathrm{CU}$ refers to a compressive-undercompressive wave pair, also known as a double wave structure. The solid line, a function of $h_{R}$, determines when an undercompressive wave (U) is selected and the dashed line, also a function of $h_{R}$, determines the intermediate height in the UC wave pair.

hyperbolic conservation law with nonconvex flux $f(h)=h^{2}$ $-h^{3}$ modeling the effect of the gravity $\left(h^{3}\right)$ and Marangoni $\left(h^{2}\right)$ forces. The form of the terms, such as $h^{3}$ for gravity, are a result of the standard depth averaging of the velocity and nondimensionalization outlined above (for details, see [1]). The nonconvex flux is particularly important because it provides a necessary condition for the existence of undercompressive waves. For a description of how nonclassical waves typically appear in equations with a nonconvex flux, see [14].

The fourth-order right-hand term in Eq. (1) models the effect of surface tension. As in [6], we neglect a second-order term modeling the component of gravity normal to the substrate, which (when included) smooths solutions. Note that if this diffusive term dominated the effects of surface tension, naturally occurring oscillations due to surface tension would be suppressed. However, observations of oscillations in physical experiments indicate that surface tension usually dominates diffusion [15].

\section{B. Riemann map for the thin-film equation}

To describe the boundary conditions for the model, we will refer to the bottom of the plate as left $(\mathrm{L})$ and the top of the plate as right $(\mathrm{R})$ as shown in Fig. 1. The film emerges from the reservoir at height $h_{L}$ and the silicon wafer is precoated with film thickness $h_{R}$. It is possible to allow $h_{L}$ $<h_{R}$, which can be achieved in experiments by partially dipping the substrate. Here, $h_{R}$ is assumed to be a thin prewetting fluid layer.

Numerical solutions of Eq. (1) exhibit a variety of single waves and pairs of waves that can emerge in a Marangonidriven film with no additional localized forcing. As described in [5], the Riemann map shown in Fig. 2 classifies the qualitatively different types of waves that emerge from a monotonic initial fluid profile with boundary conditions

$$
h(-\infty, 0)=h_{L}, \quad h(\infty, 0)=h_{R} .
$$

The map is named for the Riemann problem for partial differential equations with specified left and right boundary 
conditions, with a jump from one value (here height) to another. To simulate solutions of such a problem, the jumps are often slightly smoothed. For the simulations presented in this paper, the Riemann initial condition is smoothed using a hyperbolic tangent function as

$$
h(x, 0)=\left(h_{L}-h_{R}\right)\left[\tanh \left(j_{0}-x\right)+1\right] / 2+h_{R},
$$

where $j_{0}$ is the initial position of the jump on the $x$ axis.

Solutions of the Riemann problem include both classical waves, such as compressive waves and rarefactions, and nonclassical waves, such as undercompressive waves [1,14]. Classical waves are defined by the Lax entropy condition; in the two-dimensional thin-film model (see [16]), films with such waves are unstable to perturbations and exhibit fingering instabilities in the direction transverse to the flow. In contrast, undercompressive waves violate the Lax entropy condition and are stable to perturbations. Undercompressive waves usually occur as the leading wave in pair with a trailing compressive wave. All of the structures found in the Riemann map of Fig. 2, including both classical and nonclassical waves, were observed in numerical simulations of Eqs. (1) and (2) found in [5] and in the physical experiments of Behringer and collaborators described in $[3,17,18]$. These waves form a basic set of solutions that will be modified by the addition of localized forcing.

In the Riemann map of Fig. 2, the axes represent the boundary conditions on the heights $h_{L}$ and $h_{R}$ for solutions of Eqs. (1) and (2). The map is defined such that each point $\left(h_{L}, h_{R}\right)$ represents a solution of Eqs. (1) and (2) for those boundary conditions and monotonic initial data. The label on each region denotes the type of wave structure that will develop: $\mathrm{C}$ stands for a compressive wave, $\mathrm{U}$ stands for an undercompressive wave, and $\mathrm{R}$ stands for a rarefaction. $\mathrm{CU}$ refers to a compressive-undercompressive (CU) wave pair, also known as a double wave structure.

In the Riemann map, the eight regions are separated by four curves. The diagonal separates the solutions with jump-up initial conditions $\left(h_{L}<h_{R}\right)$ from those with jumpdown initial conditions $\left(h_{L}>h_{R}\right)$. The horizontal line in the map results from an inflection point in the nonconvex flux function $f(h)=h^{2}-h^{3}$. The remaining two curves are derived from calculations based on the theory of LeFloch and Shearer, in which classical waves are selected, and undercompressive waves occur when no classical wave is available as a solution.

The theory of LeFloch and Shearer is fully described in [4,5]. We present a brief outline here. The curves in the Riemann map are computed by seeking traveling-wave solutions of Eq. (1) with speed $s, h(x, t)=\tilde{h}(x-s t)$, and writing the resulting third-order ordinary differential equation (ODE) as a system of three first-order ODEs. In the language of dynamical systems, traveling-wave solutions are heteroclinic orbits from an unstable equilibrium at $\left(h, h^{\prime}, h^{\prime \prime}\right)=\left(h_{L}, 0,0\right)$ to a stable equilibrium at $\left(h, h^{\prime}, h^{\prime \prime}\right)=\left(h_{R}, 0,0\right)$. Solutions lie in the intersection of the unstable manifold centered at $\left(h_{L}, 0,0\right)$ and the stable manifold centered at $\left(h_{R}, 0,0\right)$. The speed $s$ for each wave can be calculated using the RankineHugoniot conditions for the jump.

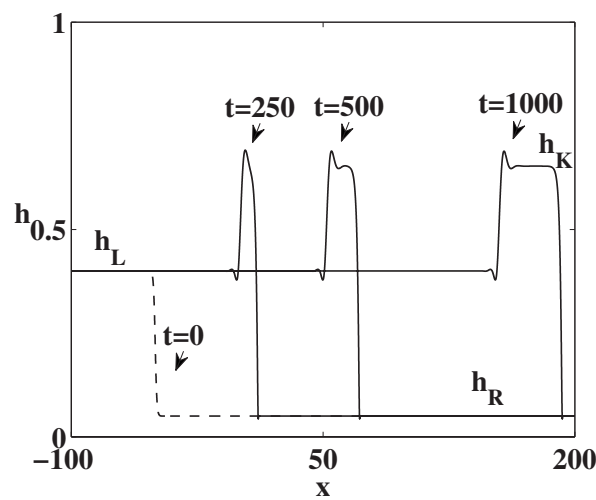

FIG. 3. Formation of a double wave structure from a monotonic initial condition with $h_{L}=0.4$ and $h_{R}=0.05$ (with a smoothed step down from $h_{L}$ to $h_{R}$ ), yielding an intermediate height of $h_{K}$ $=0.653$.

The values for the solid curve in Fig. 2 correspond to threshold values beyond which there is no longer an intersection of the unstable manifold associated with $h_{L}, W^{U}\left(h_{L}\right)$, and the stable manifold associated with $h_{R}, W^{S}\left(h_{R}\right)$. The values for the dashed curve in Fig. 2 correspond to two simultaneous intersections: one between the unstable manifold $W^{U}\left(h_{L}\right)$ and the stable manifold associated with an intermediate equilibrium $W^{S}\left(h_{M}\right)$ and a second intersection between $W^{U}\left(h_{M}\right)$ and $W^{S}\left(h_{R}\right)$. The relevance of this curve in the context of this paper is that the value on this curve as a function of $h_{R}$ selects the intermediate height when a double wave structure develops. For example, when $h_{L}=0.4$ and $h_{R}$ $=0.05$, the intermediate height $h_{K}=0.653$ develops, as predicted in the map and shown in Fig. 3. More exposition on this theory, including details of these calculations, can be found in [4,5]. An interesting aspect of this analysis is that while it is based on theory for solutions of hyperbolic conservation laws, it applies to solutions of the parabolic unforced PDE (1), as long as the initial data are monotonic.

In this paper, we will focus on a particular region of the Riemann map. Consider the transition between the lower regions connected by the arrow in Fig. 2. For example, fix a value of the prewetting height $h_{R}<0.3$ and choose moderate values of the upstream height $h_{L}$ such that $h_{L}>h_{R}$. As $h_{L}$ increases, the theory predicts a transition from a single classical wave to an undercompressive-compressive (UC) wave pair that can be observed in numerical simulations [5]. This double wave structure is composed of a trailing slower compressive wave and a leading faster undercompressive wave. The same transition from single compressive wave to double wave structure can be achieved in physical experiments (without localized forcing) by decreasing the inclination angle. At lower inclination angles, the film emerges from the reservoir in a thicker layer which corresponds to a larger value of $h_{L}$ [19]. In the next section we describe how Eqs. (1) and (2) are modified with the addition of localized forcing.

\section{Model with localized forcing}

Localized forcing provides a mechanism for additional control of the film flow. In physical experiments, it can be 
implemented by directing an infrared laser at the film at the center of the domain (initiated before the film has advanced up the substrate). To create a line of forcing, the laser can be translated quickly back and forth across the film, at a fixed position $x$ while varying $y$ (see the coordinates of Fig. 1). This experimental technique approximates the steady forcing we will incorporate into the one-dimensional model. Sur et al. observed in $[3,18]$ that such forcing impedes the advancing film and, if strong enough, creates a dry region. Sur et al. also noted that strong forcing causes some fluid to move back down the substrate (left) even when the Marangoni force imparted by the temperature gradient at the substrate is strong enough to move the film upward (right). We will discuss numerical results for this type of solution in the next section. As with the unforced model, the height $h(x, t)$ is assumed to be positive, which limits the range of forcing to values that do not cause dewetting of the substrate.

Localized forcing is modeled as an additional term in Eq. (1):

$$
\frac{\partial h}{\partial t}+\frac{\partial}{\partial x}\left(h^{2}-h^{3}\right)=M \frac{\partial}{\partial x}\left(h^{2} \frac{d \theta}{d x}\right)-\frac{\partial}{\partial x}\left(h^{3} \frac{\partial^{3} h}{\partial x^{3}}\right) .
$$

This is the PDE we will consider for the remainder of the paper. The forcing function is usually taken to be a Gaussian centered at $x=0$,

$$
\theta(x)=\left(\frac{1}{\sqrt{2 \pi}}\right) e^{-x^{2} / 2} .
$$

The nondimensional coefficient $M$ can be thought of as a parameter to control the level of forcing or, in physical terms, $M=\frac{I}{\tau L}$ is the ratio of temperature variations due to localized heat intensity $(I)$, uniform temperature gradient $(\tau)$, and characteristic length scale $L$ [6]. In [6], the intensity $I$ was not calibrated to the experiment, and thus $M$ was employed as a qualitative measure of the effect of localized forcing.

The term region of forcing will be used to refer to the interval on the $x$ axis for which the forcing function has magnitude greater than the grid spacing. Unless otherwise noted, the spatial grid size used in computations is $10^{-2}$. All of the chosen forcing functions decay at least exponentially; therefore, even though they do not have finite support, the forcing is effectively localized to a small interval. For instance, for the Gaussian forcing, this region is contained within the interval $[-2,2]$. Thus, solutions of Eq. (3) outside this region are approximately solutions of Eq. (1). To simulate solutions of Eq. (3), we use a standard Crank-Nicolson finite difference scheme with an adaptive implicit time step [20]. In practice, a time step of $10^{-2}$ is effective. The method uses centered spatial differences and is second-order accurate in space and time.

\section{SIMULATIONS WITH CONSTANT INITIAL CONDITIONS}

Haskett et al. identified two steady-state solutions of Eq. (3) for constant initial data with a bifurcation between the steady states occurring at a critical value of the forcing pa-
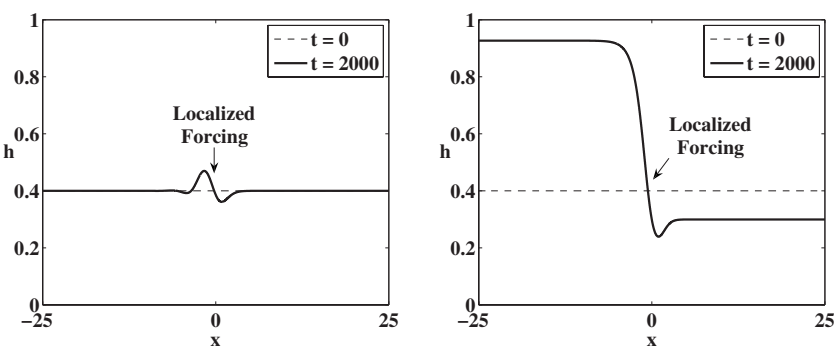

FIG. 4. Steady-state solutions of Eq. (3). Fluid profiles approach a type I solution for $M=0.5<M_{C}\left(h_{L}=0.4\right) \approx 1.16$ (left) and a type II solution for $M=1.5>M_{C}\left(h_{L}=0.4\right) \approx 1.16$ (right). The initial fluid profile $h(x, 0)=0.4$ is a black dashed line in each plot.

rameter $M_{C}$. Type I solutions are homoclinic orbits resulting from PDE simulations with weak forcing, $M<M_{C}$. Type II solutions are heteroclinic orbits resulting from strong forcing $M>M_{C}$. For constant initial profiles, as the upstream film thickness $h_{L}$ increases, the minimum level of forcing required to produce a type II solution decreases [6]. Recall that $h_{L}$, the thickness where the film emerges from the reservoir at the bottom of the substrate, can be increased by lowering the inclination angle of the substrate.

Examples of both types of steady-state solutions are illustrated in Fig. 4 (solid lines) along with the initial condition (dashed lines). In the plots, the simulation has been run long enough that transient wave structures have moved outside the domain of interest, leaving only the steady-state fluid profile. The evolution of the transients will be discussed in Secs. II A and III. As expected from the steady-state asymptotics considered in [6], numerical experiments confirm that for type I solutions, the shape of the perturbation near the forcing region is a direct consequence of the choice of forcing function and to leading order mimics the shape of the derivative of that function, $\theta^{\prime}(x)$.

In the next section we focus on transient structures that evolve to the steady-state solutions of Fig. 4. In this context the steady-state solution is viewed as an inner solution, with the transient waves of the outer solution eventually leaving the domain of interest. We will explain how the structure of the transient outer solution connecting the inner solution to the boundary data is related to the predictions of the Riemann map (Fig. 2).

\section{A. $\boldsymbol{N}$ waves from constant initial conditions}

The term " $N$ wave" refers to a set of waves comprised of two compressive shocks separated by a rarefaction [11]. While $N$ waves are not usually noted as solutions of the thin-film equation, they are well-known solutions of both the inviscid Burgers equation, a hyperbolic conservation law, and the viscous Burgers equation, which includes an additional parabolic regularizing term [21]. For these equations, $N$ waves are generic behavior arising from zero-mean perturbations. The primary goal of this section is to explore transient $N$ waves in numerical solutions of Eq. (3) with constant initial fluid profile $h(x, 0)=h_{0}$ and Gaussian forcing. We relate the evolution of the solutions to the theory for the Riemann map described in Sec. I B. 

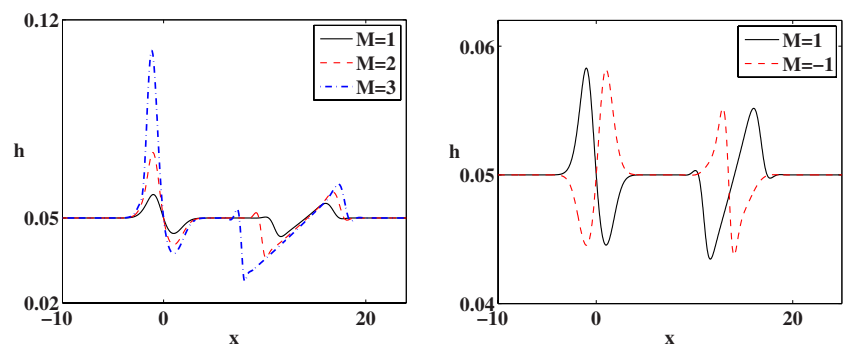

FIG. 5. (Color online) Fluid profile at time $t=150$, showing $N$ waves (two shocks with a rarefaction between them) produced from a constant initial height $h_{0}=0.05$ for a range of forcing parameter $M<M_{C}$ (left). The $N$ waves have an amplitude comparable to that of the disturbance of the film due to localized forcing at $x=0$. Negative values of $M$ produce two rarefactions with a shock between them (dashed line on right).

For Gaussian forcing with $M>0$ and a constant initial fluid profile $h(x, 0)=h_{0}$, the downstream transient that appears in the evolution of the type I steady state is an $N$ wave, as shown in Fig. 5 (left). Restricting $h_{0}<2 / 3$ ensures that transients flow downstream. One notable feature of the $N$ waves in Fig. 5 (left) is that the magnitudes of the jump heights vary with the forcing strength $M$. Although for moderate $M$ the heights increase approximately linearly with $M$, the left shock height grows more dramatically with increasing $M$ than the right shock, a relationship shown in Fig. 6 . This reflects an asymmetry in the type I steady state, which qualitatively assumes the shape of $\theta^{\prime}(x)$, but has a much more pronounced peak of fluid behind the forcing for large $M$ than its corresponding valley in front of the forcing. This asymmetry is a result of the temperature gradient, which drives fluid up the incline.

As the $N$ wave flows up the incline (right), the rarefaction length increases as the shocks separate, and each shock decays. The speed of each shock is approximated by the Rankine-Hugoniot conditions, by considering the right (left) shock to be a jump from the maximum (minimum) height of the $N$ wave to $h_{0}$. Note that this approximation ignores the effect of the fourth-order term. The speed of the leading shock converges rapidly to the predicted speed once the wave has exited the forcing region (shown in Fig. 7). In

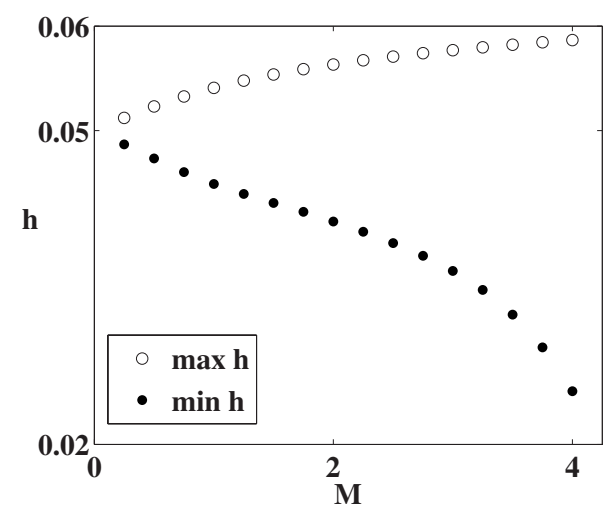

FIG. 6. Variation of $N$-wave maximum and minimum height as a function of the forcing parameter $M$ at a fixed time $t=500$. The relationship is approximately linear for $M<2$.

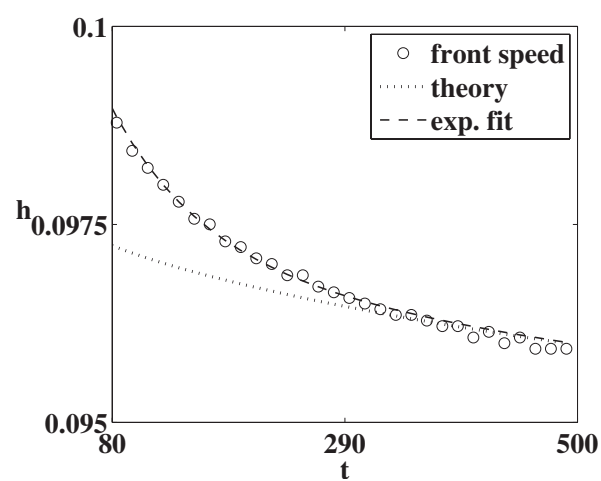

FIG. 7. Convergence of the $N$-wave front shock to the predicted speed. Note that there is some variation at large times $(t>400)$ as the shock decays and becomes less well defined. The grid spacing used here is $10^{-3}$ in order to resolve the early difference between theory and simulation, with the $N$ wave evolved from constant initial height $h_{0}=0.05$ and $M=1$. The dashed line is the speed predicted by the Rankine-Hugoniot conditions, and the dotted-dashed line is a fit of the difference between the data and theory to exponential decay given by $\exp (-0.0115 t-5.485)$. The maximum norm of the difference between approximation and data is $1.5 \times 10^{-4}$.

addition, when wave has left the forcing region, its height decays (Fig. 8) according to the expected decay rate of $O\left(t^{-1 / 2}\right)$ for an $N$ wave from hyperbolic theory [11].

\section{B. Transient formation and evolution}

Downstream transients are formed in three stages. First, perturbations are created by the forcing. Second, the perturbations are swept downstream and exit the forcing region. Finally, the transient perturbations merge according to their relative speeds to produce a profile that travels downstream as it decays. Some transients significantly decay before emerging from the forcing region, and thus may not be visible.

Initially, the perturbation for a constant initial condition $h_{0}$ and forcing function $\theta$ is approximated by

$$
h(x, t) \approx h_{0}+M h_{0}^{2} \theta^{\prime \prime}(x) t,
$$

which is a zero-mean perturbation because the forcing $\theta$ is localized [22]. The approximation agrees well with numeri-

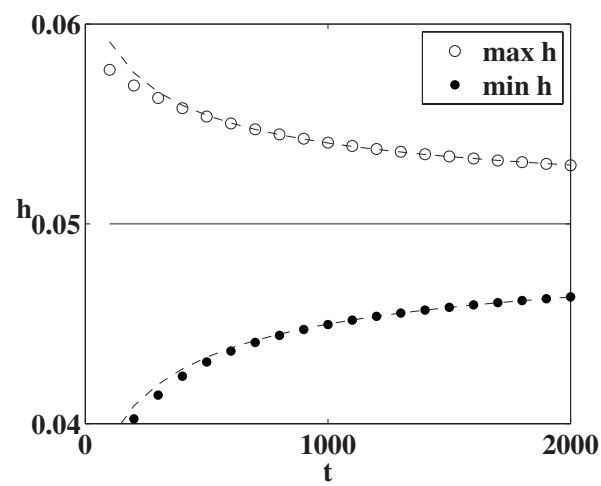

FIG. 8. The maximum and minimum heights of the fluid as it decays. The dashed lines are fits to $(a x+b)^{-1 / 2}$, indicating the $O\left(t^{-1 / 2}\right)$ rate of decay expected for the height of an $N$ wave. For the maximum height the fit parameters are $a=54.6$ and $b=6.60 \times 10^{3}$, and for the minimum height, $a=34.6$ and $b=5.13 \times 10^{3}$. 


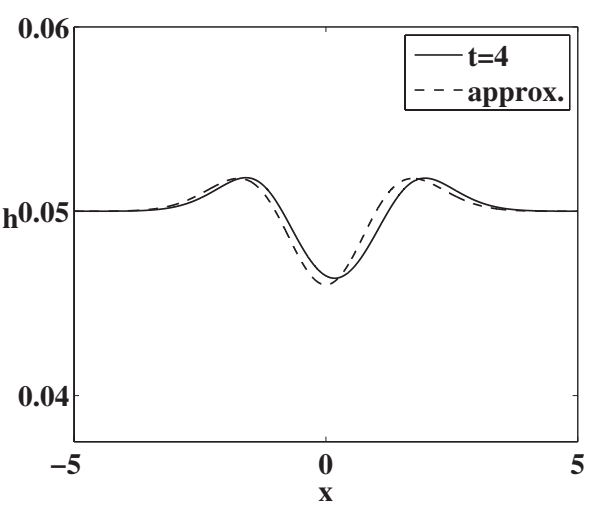

FIG. 9. For $M=1$ and $h_{0}=0.05$, a comparison of the fluid profile at an early time $t=4$ (solid line) to the approximation $h(x, t) \approx h_{0}$ $+M h_{0}^{2} \theta^{\prime}(x) t$ (dashed line).

cal simulations as shown in Fig. 9 and is valid for small $t$, when the forcing term is dominant and before the perturbations are swept significantly downstream. Equation (4) can be deduced analytically from the PDE by considering the asymptotic expansion $h(x, t)=h_{0}+h_{1}(x) t+O\left(t^{2}\right)$. The qualitative shape of the downstream transients is determined by this perturbation.

We next consider the evolution of transients about a point $x=x_{0}$ where the forcing gradient has a maximum. When $\theta^{\prime \prime}\left(x_{0}\right)=0$, the second term in Eq. (4) is zero and the film is unperturbed. Generally, $\theta^{\prime}\left(x_{0}\right)$ assumes opposite signs on each side of $x_{0}$, and the height is increased or decreased according to Eq. (4). The perturbation upstream of $x_{0}$ will be trapped and build up if the forcing is strong enough, whereas the downstream perturbation will be swept away from $x_{0}$.

The downstream transients, therefore, arise from peaks (valleys) that form to the right of the maxima (minima) of the forcing gradient and evolve into shock-rarefaction pairs. The sharpness of the resulting shock depends on the strength of the forcing, and the mass of the resulting structure is determined by the size of the perturbation. This evolution is predicted by the Riemann map in Sec. I B, noting that in the Riemann map, for small $h_{L}$ and $h_{R}$, the diagonal $h_{L}=h_{R}$ separates a region corresponding to rarefactions from the one for compressive shocks. Therefore, a small perturbation from constant height $h_{0}<1 / 3$ will evolve into a shock-rarefaction pair.

\section{Gaussian forcing}

In [6], the authors considered steady-state solutions for Gaussian forcing since it is a reasonable ansatz for localized forcing, such as laser heating. In the next sections we analyze transient structures for the same forcing function, then compare the results to forcing functions that more clearly illustrate aspects of the early-time solutions.

In Fig. 10 we show the evolution of an $N$ wave from Gaussian forcing. Initially the forcing creates a perturbation. Then additional fluid gathers behind the forcing while the rightmost peak moves to the right, sharpening as it leaves the forcing region (Fig. 10, left) to form a leading shock and a rarefaction consistent with the predictions of the Riemann map in Fig. 2. A second shock, a negative perturbation con-
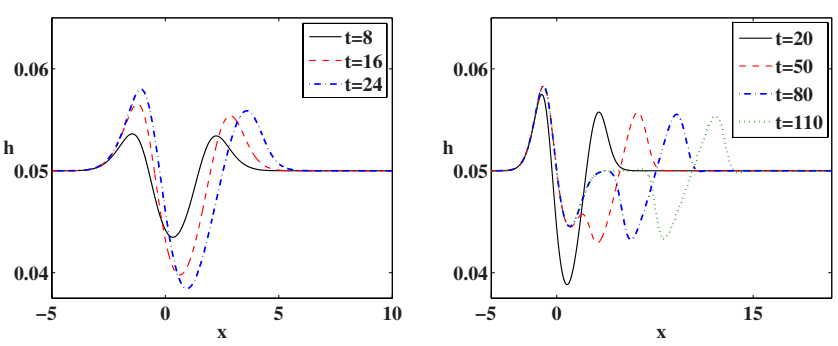

FIG. 10. (Color online) The evolution of the fluid profile at an early time. Fluid gathers behind the forcing while the rightmost peak is transported downstream. The rightmost shock sharpens as it leaves the forcing, and the second shock subsequently emerges from the forcing as well.

nected to the rarefaction behind the first, then emerges from the forcing. The $N$ wave finally exits the forcing region, leaving the expected steady state about $x=0$ (Fig. 10, right).

\section{Rectangular forcing}

To more clearly observe transient formation from multiple inflection points, we consider a smoothed rectangular forcing function as shown in Fig. 11 (left). This forcing function is given by

$$
\theta(x)=(2 w)^{-1}\{\tanh [r(x+w / 2)]-\tanh [r(x-w / 2)]\},
$$

where $w$ is the approximate width (the forcing region is contained in $[-w / 2-1, w / 2+1])$ and $r$ controls the sharpness of the forcing. Here, the regions of rapid change in the forcing function are separated, producing two nearly disjoint opposite peaks which evolve separately. Initially, a peak and the corresponding valley evolve around each inflection point of $\theta$ (according to the approximation) and the rightmost perturbation is swept downstream, evolving into a shockrarefaction pair. The small oscillation in the figure between the shocks is itself a small shock which does not appear in the Gaussian case. Note that for the left shock-rarefaction pair, the rarefaction extends slightly above the height $h_{0}$, leading into a small positive perturbation (this is due to fourth-order effects). The right shock-rarefaction pair has a similar negative perturbation. As the rarefactions approach each other, these perturbations create the small shock visible as the waves combine.
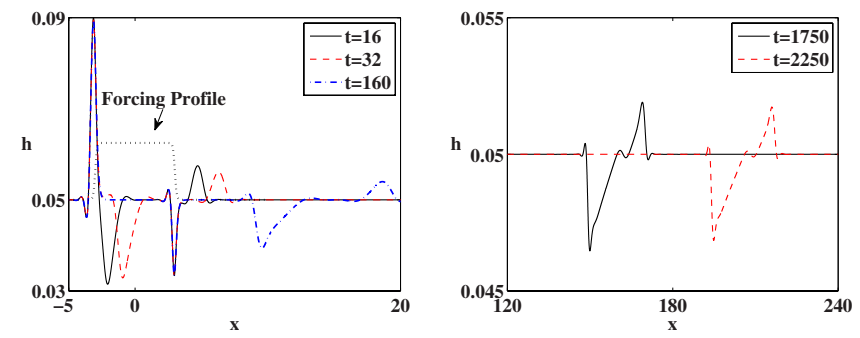

FIG. 11. (Color online) Evolution from a smoothed rectangular forcing with $M=2$, in which the formation of the positive and negative perturbations is separated. The shock-rarefaction pairs eventually merge to form an $N$ wave (with the small oscillation between the shocks still visible in the right plot). 

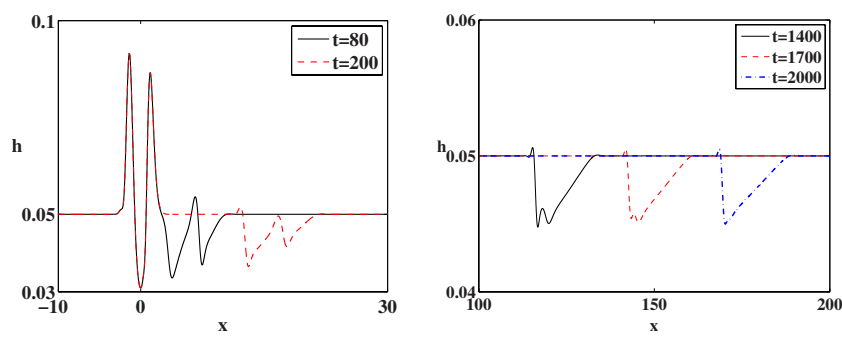

FIG. 12. (Color online) Wave structure from the forcing $\theta(x)=$ $-x e^{-x^{2}}$ with $M=1.5$. The transient structure mirrors the forcing, and the central shock decays rapidly. Eventually, the two shocks merge (right) to form a single shock-rarefaction pair.

\section{Forcing with additional inflection points}

We next consider a forcing function that illustrates how multiple inflection points create shock-rarefaction pairs that merge. In general, after leaving the forcing region, waves merge according to their relative speeds. Here, we consider an odd forcing function,

$$
\theta(x)=-x e^{-x^{2}}
$$

with forcing region $[-2.5,2.5]$. (This happens to be the derivative of the Gaussian forcing function, but this is not significant to the result.) This forcing function produces two shock-rarefaction pairs (Fig. 12, left) with a transient between them to form a "W" shape. The rightmost shock is eventually overtaken (Fig. 12, right) by the faster left shock, forming a single shock-rarefaction pair.

\section{Triangular forcing}

The purpose of considering triangular forcing is to emphasize that the appearance of an $N$ wave is not sensitive to the choice of forcing as long as $\theta^{\prime}(x)$ has the same extrema as the Gaussian forcing. That is, in general, $N$ waves will arise from any localized forcing function $\theta(x)$ for which $\theta^{\prime}(x)$ has a local maximum (greater than $h_{0}$ ), followed by a local minimum (less than $h_{0}$ ), which-respectively-produce rarefaction-shock (positive perturbation) and shockrarefaction (negative perturbation) pairs that merge and form an $N$ wave. For example, we considered the triangular forcing function given by

$$
\theta(x)=[w g(w / 2)]^{-1}[g(x+w / 2)+g(x-w / 2)-2 g(x)],
$$

where $g(x)=\log \cosh (r x)$ is a smooth approximation for $r|x|$, with $r$ fixed, and $w$ is the approximate width (the forcing region is slightly larger than $[-w / 2, w / 2]$ ). Using the triangular forcing function above with $w=6$ and $r=8$ results in a similar $N$ wave (see Fig. 13). The shocks are steeper because the initial perturbation, close to the shape of $\theta^{\prime \prime}$, is much sharper than the Gaussian forcing due to the rapid change in $\theta^{\prime}$ at the corners of the initial triangle.

The process of merging waves also explains the rarefaction-shock-rarefaction structure that results from Gaussian forcing with $M<0$. As in the $N$-wave case, two shock-rarefaction pairs are produced by the forcing, but the
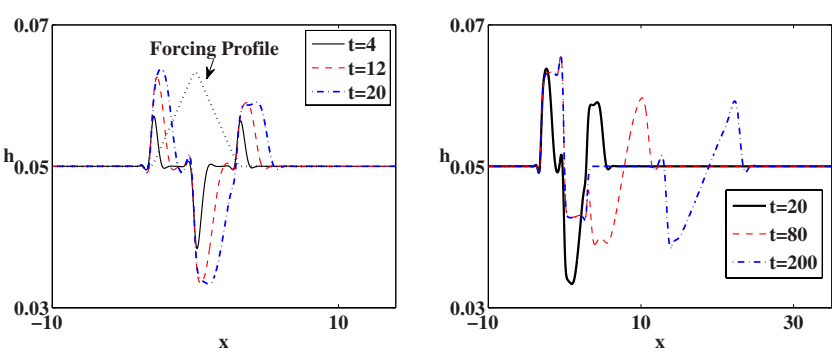

FIG. 13. (Color online) Evolution from a smoothed triangleshaped forcing with $M=1$. The result is an $N$ wave similar to that produced by Gaussian forcing. The rapid change in the forcing derivative at the corners yields a sharper $N$ wave than for the corresponding $M=1$ Gaussian case.

left shock in the $M<0$ is faster than the right shock, and so the two shocks merge to form a single shock between the two rarefactions, as shown in Fig. 5 (right).

\section{Observing $N$ waves in experiments}

$N$ waves have not been noted in previous physical experiments on Marangoni and gravity-driven thin liquid films. This is likely because in experiments, the amplitude of the $N$ wave would be relatively small. Since previous experiments focused on type I and II steady-state solutions, the $N$ waves may have been overlooked as they were over-run by the larger waves. For instance, Fig. 14 shows a compressive shock forming the downstream height $h_{B}$ in a type II steadystate solution. Its speed is faster than that of the $N$ wave (with a speed of approximately 0.24 compared to 0.09 for the $N$ wave), and so the $N$ wave is over-run. This is typical behavior of the system in the case of a type II solution with Riemann initial data $h_{L}>h_{R}$, which is explored more fully in Sec. III. There is also some difficulty in producing $N$ waves experimentally. $N$ waves are only visible as downstream transients to type I solutions, and consequently $M$ must be small enough so that $M<M_{c}$ but also large enough to make the $N$-wave magnitude significant without dewetting the substrate. Despite these potential challenges, future experiments could be conducted to investigate the development of $N$ waves, as well as the applicability of localized forcing as a microfluidic valve for thin films.
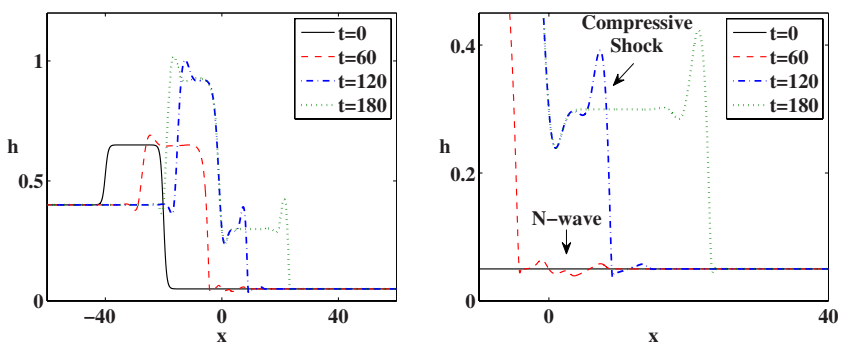

FIG. 14. (Color online) Over-run of the $N$ wave by a faster compressive wave. The downstream compressive wave is the downstream transient for a type II solution dictated by the left boundary condition $h_{L}$, whereas the $N$ wave is formed from the effective constant initial condition at $h_{R}$ in the forcing region. This type II solution is discussed in Sec. III. 

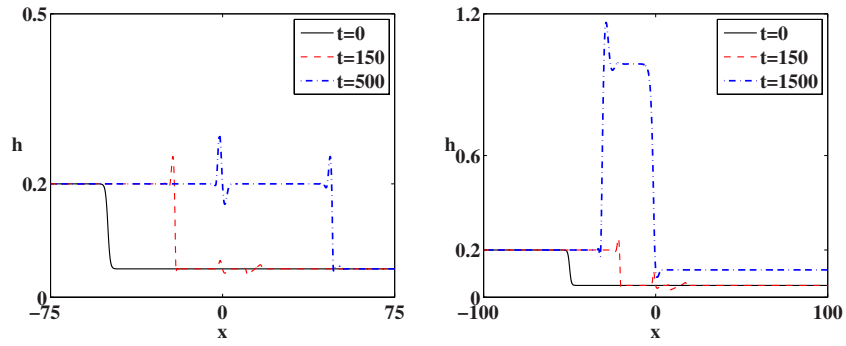

FIG. 15. (Color online) Single compressive waves and Gaussian forcing. On the left, $h_{L}=0.2, h_{R}=0.05$ with weak forcing $M=1.5$ $<M_{C}$ leads to a type I solution. On the right, $h_{L}=0.2, h_{R}=0.05$ with strong forcing $M=3.0>M_{C}$ leads to a type II solution.

\section{SIMULATIONS WITH CONTROLLED NONMONOTONIC INITIAL CONDITIONS}

Up to this point we have only discussed solutions arising from a constant initial film height. Next we describe solutions that emerge from nonmonotonic initial conditions and the Gaussian forcing function. As described in Sec. II A, both type I and type II steady-state solutions of Eq. (3) emerge from a constant initial film (selected by choosing the forcing parameter $M$ ). When the initial film profile is monotonic but not constant, such as a monotonic jump up or down from $h_{L}$ to $h_{R}$, solutions of the unforced PDE (1) include classical compressive waves, classical rarefactions, and nonclassical undercompressive waves. The types of waves that emerge from monotonic initial data depend on the choice of boundary data $h_{L}$ and $h_{R}$; for each choice of $\left(h_{L}, h_{R}\right)$ the resulting wave is denoted in the Riemann map of Fig. 2. The shape of early transients is determined by the shape of the initial profile.

We restrict our study to $h_{L}>h_{R}$ with $h_{L} \ll 2 / 3$ and small $h_{R}$. If the initial boundary heights $\left(h_{L}, h_{R}\right)$ are chosen in the $\mathrm{C}$ (compressive) region of the Riemann Map (Fig. 2), a single compressive wave profile will evolve. In this case the steadystate solutions are the same as those for constant initial profile. Figure 15 contains two simulations with the same compressive wave $\left(h_{L}=0.2, h_{R}=0.05\right)$ moving through a forcing region above and below $M_{C}\left(h_{L}=0.2\right)$. In Fig. 15 (left) with $M=1.5$ the steady-state solution is type I, and in Fig. 15 (right) with $M=3.0$ the steady-state solution is type II. Note that $M=3.0$ is below the critical forcing for a height of 0.05 , but the solution is type II because the critical forcing depends on $h_{L}$ rather than $h_{R}$.

On the other hand, if the initial boundary heights $\left(h_{L}, h_{R}\right)$ are chosen in the $\mathrm{CU}$ region of the Riemann map (Fig. 2), a nonmonotonic double wave profile with a leading undercompressive wave and a trailing compressive wave will evolve. The goal of this section is to discuss how this nonmonotonic structure interacts with the forcing region. In time the double wave structure becomes wider since the leading undercompressive wave is faster than the trailing compressive wave. For the monotonic initial condition with a single jump down, as we vary $h_{L}, h_{R}, M$, and the initial location of the jump, the degree to which the $\mathrm{CU}$ structure will have developed varies as well.

We wish to control the width (and overall shape) of the structure as it enters the forcing region to probe the effect of

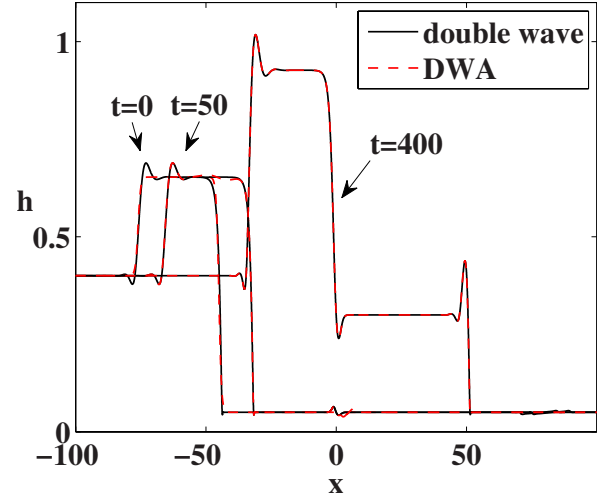

FIG. 16. (Color online) Comparison of a double wave structure (as in Fig. 3), evolved from a jump at $x=-250$ for 850 units of time before the labeled $t=0$, with a DWA was chosen to match the nucleated and boundary heights. The shapes become nearly identical as the solutions evolve. The forcing strength is $M=1.5$.

varying parameters. Thus, to regularize the initial data for the simulations, we construct an initial condition that approximates the (nonmonotonic) double wave structure from the Riemann map of Fig. 2 that evolves from a monotonic initial condition with $h_{L}>h_{R}$.

\section{A. Double wave approximation}

We have noted that when a monotonic initial condition is varied, the time and location of evolution of the double wave structure also vary, which provides less control than is desirable. In addition, evolution of the double wave structure is expensive computationally (requiring enough time and space to move beyond the transient regime). In order create an efficient and controlled initial condition with variable width and height, we artificially construct an initial profile that mimics a double (CU) wave in shape and mass. For convenience, we call this a double wave approximation (DWA).

Consider a monotonically nonincreasing initial condition with $h_{L}>h_{R}$ chosen in the region of Fig. 2 labeled CU. Upstream of the forcing, the traveling-wave solution evolves as if it were a solution of the unforced Eq. (1) until it reaches the forcing region. As expected from the Riemann map, a double wave structure composed of a compressiveundercompressive wave pair develops [13,23]. The height of the film $h_{K}\left(h_{R}\right)$ between the wave pair is given by the point $\left(h_{K}, h_{R}\right)$ on the kinetic relation curve in Fig. 2 and so is determined by $h_{R}$ when it exists.

Figure 16 contains a comparison between a double wave structure and a DWA. The double wave structure has evolved for 850 time units from an initial condition with jump at $x$ $=-250$. The DWA parameters have been chosen so that the integrated area closely matches the actual solution (a difference of 0.0038), and so that the shocks that evolve are as close as possible to those evolved in a double wave structure. The capillary ridge not present in the DWA at $t=0$ (connecting the left shock to the height $h_{K}$ ) has formed by $t=50$, and the "shocks" defined in the DWA as smoothed jumps remain in the appropriate positions as the solution evolves. The absolute maximum height difference between the two solutions 
TABLE I. Relevant heights for a double wave structure interacting with forcing, illustrated in Fig. 17.

\begin{tabular}{lcc}
\hline \hline Label & Meaning & Dependence \\
\hline$h_{L}$ & Upstream boundary & Fixed \\
$h_{R}$ & Downstream boundary & Fixed \\
$h_{K}$ & Central height of UC pair & $h_{R}$ \\
$h_{C}$ & Critical height for type II & $M$ \\
$h_{A}$ & Upstream type II height & $M$ \\
$h_{B}$ & Downstream type II height & $M$ \\
\hline \hline
\end{tabular}

is 0.1 at $t=0,0.0082$ at $t=50$, and 0.0051 at $t=150$. This measurement excludes the downstream transient in the DWA, which has already exited the domain. This difference occurs near the shocks, yet when the solutions interact with the forcing, they are similar enough to produce essentially the same behavior. The maximum difference at $t=400$, the last time shown in Fig. 16, is 0.01, with the largest error occurring in the upstream shock because of its horizontal offset. This suggests that the DWA is an appropriate approximation for conveniently evolving double wave structures. Note that the error at late times is on the order of the grid size, $\Delta x=0.01$.

As the DWA collides with the forcing, the solution depends on both $M$ and the boundary heights. The relevant heights are summarized in Table I and illustrated in Fig. 17. The choice of $M$ determines three heights: the type II upstream height $h_{A}$ and downstream height $h_{B}$, as well as the critical height $h_{C}$. The heights $h_{A}$ and $h_{B}$ are related by the flux balance $h_{A}^{2}-h_{A}^{3}=h_{B}^{2}-h_{B}^{3}$ with nontrivial root

$$
h_{B}=\frac{1}{2}\left[1-h_{A}+\sqrt{1+2 h_{A}-3\left(h_{A}\right)^{3}}\right] .
$$

The critical height $h_{C}$ is defined such that a constant initial condition $h_{0}>h_{C}$ will produce a type II solution, whereas $h_{0}<h_{C}$ will produce a type I solution. Table II contains an example of how the relevant parameters vary with $M$. For type II solutions, $f\left(h_{B}\right)$ is the flux through the forcing region

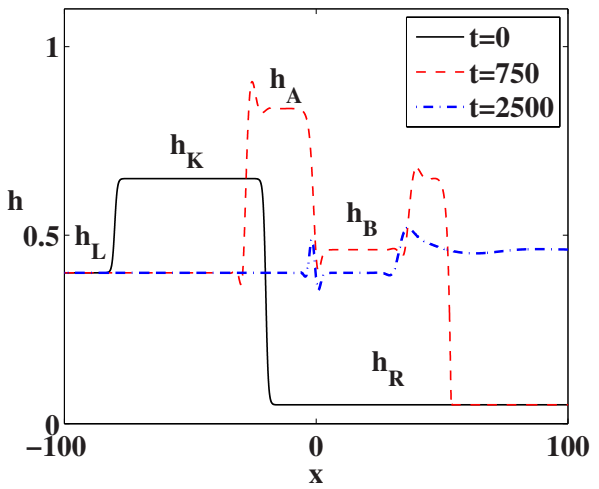

FIG. 17. (Color online) The DWA of Fig. 16 interacts with localized forcing, with relevant heights labeled. Here, $M=0.6$ and $h_{C}(M) \approx 0.5$ is the critical height. The other values are $h_{R}=0.05$, $h_{L}=0.4, h_{K}=0.65$ for the double wave structure and $h_{A}=0.84, h_{B}$ $=0.46$.
TABLE II. The heights $h_{A}, h_{B}$, and $h_{C}$ for the values of $M$ used in this section. The values $h_{A}, h_{B}$ as functions of $M$ and $M_{c}(h)$, the functional inverse of $h_{c}(M)$, can be found in [6].

\begin{tabular}{ccccl}
\hline \hline$M$ & Forcing type for $h_{L}=0.4$ & Type II $h_{A}$ & Type II $h_{B}$ & $h_{C}$ \\
\hline 0.6 & Weak (steady-state type I) & 0.84 & 0.46 & 0.5 \\
1.0 & Moderate (bistable) & 0.89 & 0.38 & 0.43 \\
1.5 & Strong (steady-state type II) & 0.93 & 0.30 & 0.36 \\
\hline \hline
\end{tabular}

and $f\left(h_{L}\right)$ is the flux into the system at the left boundary. Recall that $h_{K}$ is the intermediate height of the double wave structure, determined by $h_{R}$. The right boundary is taken to be the small prewetting height $h_{R}=0.05$, which determines $h_{K}=0.653$ for the DWA. The choice $h_{L}=0.4$ and a DWA create a transient flow greater than the boundary flux set by $h_{L}$. Figure 17 provides an illustration of how the heights in Table I are related.

In [6] there is mention of a region of bistability and a call for further investigation of this phenomenon. In fact, there are three regimes, given a DWA as an initial condition, determined by the forcing strength $M$ and the left boundary $h_{L}$. Weak and strong forcing leads to type I and II steady-state solutions consistent with solutions from constant initial conditions. However, for weak forcing, a transient type II solution can occur and for moderate forcing close to $M_{C}$ (the bistable region of [6]) the results are even more complicated. We describe simulations for each of these regimes below and the parameter ranges required for each solution.

\section{B. Type I solution from a DWA and weak forcing}

In the first case we maintain weak forcing such that $M$ $\ll M_{C}$. If $h_{K}, h_{L}<h_{C}$, then the incoming fluid passes through the forcing region without significant deformation (Fig. 18). However, a spike in height persists after the mass of fluid in the DWA has flowed through the forcing region, corresponding to the eventual formation of the type I steady state. The fluid will be transmitted through the forcing region even if $h_{K}>h_{B}$ (as in Fig. 18), which emphasizes the difference in

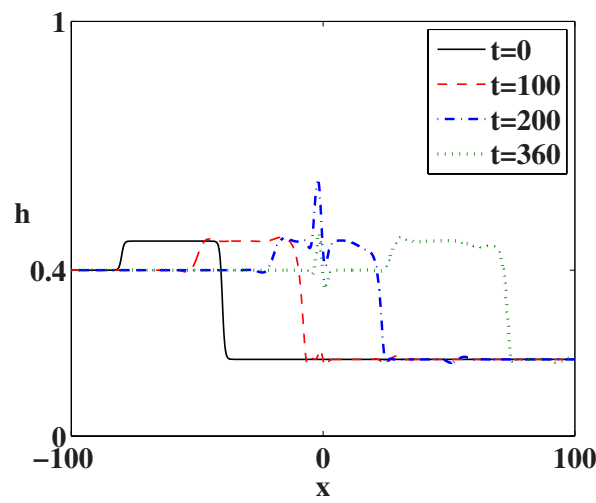

FIG. 18. (Color online) DWA with $h_{R}=0.185$ colliding with forcing at $M=0.6$. The height $h_{K} \approx 0.47$ satisfies $h_{B}<h_{K}<h_{C}$ since $h_{B}=0.46$ and $h_{C}=0.5$. The wave passes through the forcing region with little deformation, leaking through the forcing at height $h_{K}$. 

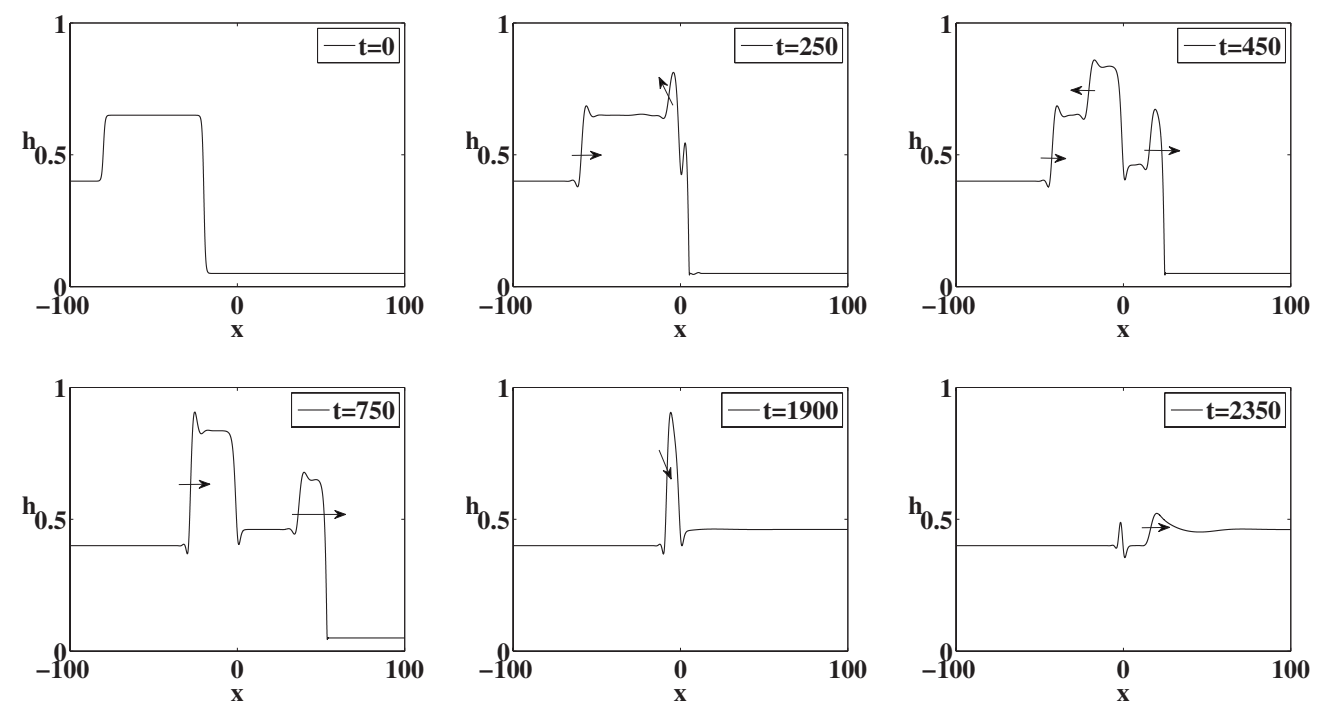

FIG. 19. Transient-induced type II leading to steady-state type I from a DWA initial condition with $M=0.6$ and a width of 60 units. Fluid passes through the forcing region at a fixed height $h_{B}=0.46$ with the remaining DWA mass gathering behind the forcing to form the upstream ridge. After the DWA is absorbed, the gathered fluid leaks through the forcing with height $h_{B}=0.46$, which finally collapses into a type I steady state.

flow for the type I and type II cases. That is, the incoming fluid must have a height greater than $h_{C}$ in order for it to collect upstream. In Fig. 18, we provide an example with $h_{K} \approx 0.47$ and $h_{B}<h_{K}<h_{C}$. In this example, $h_{R}$ is not 0.05 because $h_{K}$ needs to be small enough to pass through the forcing region.

\section{Transient type II leading to type I solution from a DWA and weak forcing}

If we maintain $M \ll M_{C}$ but choose $h_{L}<h_{B}, h_{C}$ such that $h_{K}>h_{C}$ then a type II solution occurs as a transient, eventu- ally collapsing to a type I solution. While fluid from the double wave structure is colliding with the forcing, it can only escape at the prescribed flux of $f\left(h_{B}\right)$ determined by $h_{K}$, and so some fluid gathers, which locally forms a type II solution. The shock from $h_{K}$ to $h_{A}$ created in the formation of the type II solution moves upstream from the forcing, while the compressive shock of the DWA, from $h_{L}$ to $h_{K}$, continues to move downstream. Figure 19 (top row) shows the formation of this transient type II structure with $h_{K}=0.65$ and $h_{A}$ $=0.84$. Once the double wave structure has been absorbed, the two shocks merge to become a single shock that is a
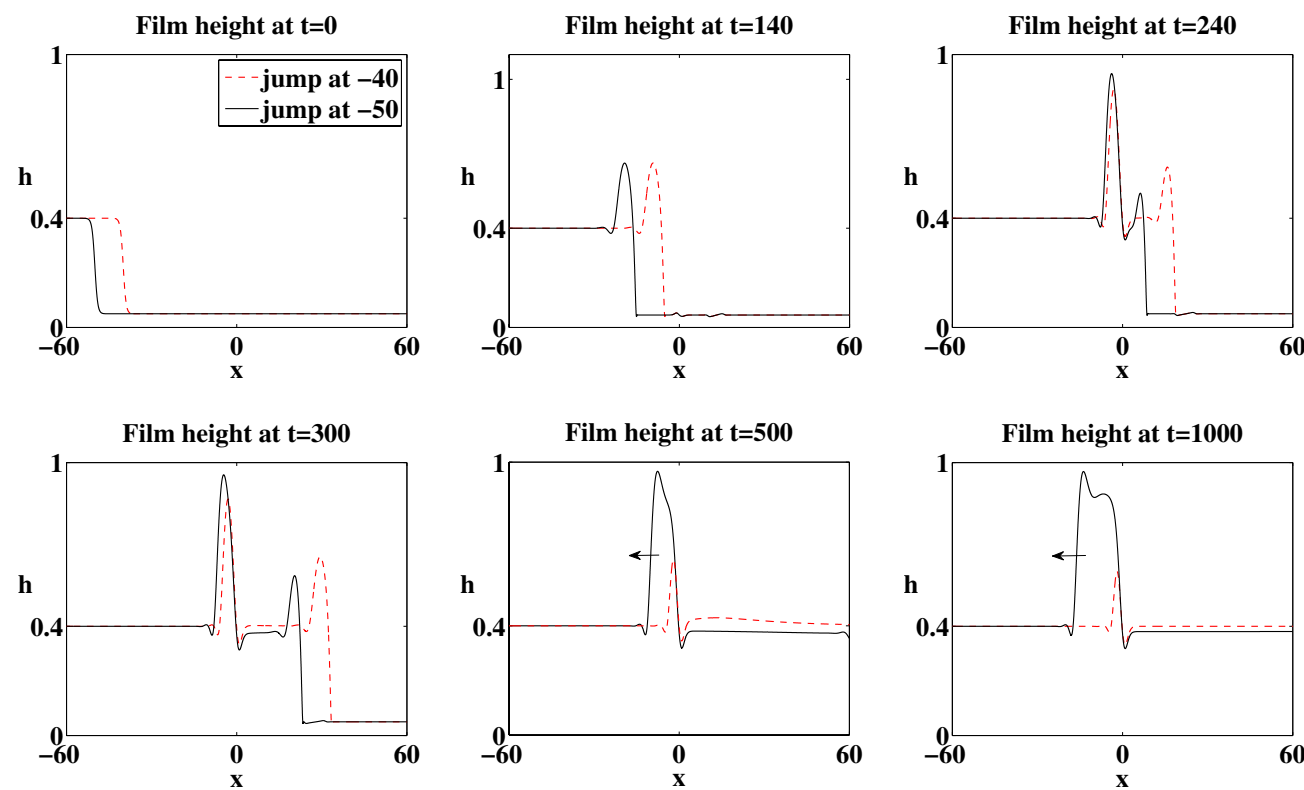

FIG. 20. (Color online) Two solutions for $M=1$ that differ only by the jump position in the initial condition. For a jump at $x=-50$ (dashed curves), the fluid in the double wave structure being formed is enough to induce a type II steady state, whereas for the jump at $x=-40$ (solid curves), the fluid leaks through the forcing to form a type I steady state. Note that for constant $h_{0}=0.4$ the solution would be type I. This simulation was also run on a larger domain $[-200,200]$ for long times to verify the stability of the type II solution. 


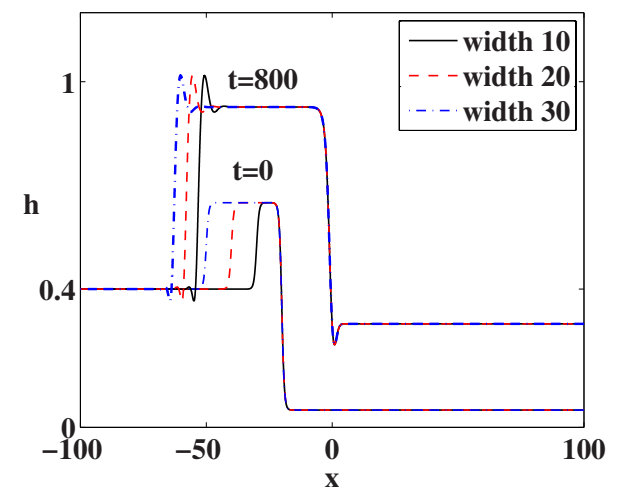

FIG. 21. (Color online) Three DWA initial conditions with varying widths, $h_{L}=0.4$, and $h_{R}=0.05$. Since $M=1.5$ is greater than $M_{c}$ for $h_{L}=0.4$, a type II solution is guaranteed, and the differing widths determine the position of the upstream shock according to the differences in mass.

jump from height $h_{L}$ to $h_{A}$ that moves to the right. The reservoir is depleted as the gathered mass of fluid leaks through the forcing region (Fig. 19, bottom row). Once the upstream ridge at $h_{A}$ is destroyed by the right-moving shock upstream of the forcing, the transient type II solution no longer exists, and so the remaining peak of fluid (bottom row, center) leaks through the forcing without being constrained to the height $h_{B}$ (bottom row, right).

\section{Type II solution from a DWA and moderate forcing}

If $h_{K}>h_{C}$ but $h_{C}>h_{L}>h_{B}$, then the double wave structure can induce a stable type II solution despite the fact that a constant initial condition $h_{0}=h_{L}$ would lead to a type I solution. The condition $h_{C}>h_{L}>h_{B}$ is a region of bistability for type I and type II solutions for constant initial condition $h_{0}=h_{L}$, as suggested in [6]. The condition $h_{K}>h_{C}$ creates a buildup of fluid behind the forcing and the local formation of a type II structure as if evolved from constant initial condition $h_{K}$ (see Fig. 20). An upstream shock is then formed connecting $h_{K}$ to $h_{A}$. After the DWA is absorbed it becomes a shock from $h_{L}$ to $h_{A}$ that continues to move to the left. The condition $h_{L}>h_{B}$ is sufficient, by the Rankine-Hugoniot condition, to guarantee that a shock formed connecting fluid at height $h_{L}$ to $h_{A}$ will flow to the left.

The width of the DWA is significant in determining whether a type II solution will be induced. The upstream shock of the type II solution forms because of constrained downstream flow and consequent upstream flow of the excess fluid. In this case, the constrained flow is transient, and so it must persist long enough for fluid to gather and the shock to form. Figure 20 shows two monotonic initial condition with $h_{L}=0.4$ and $h_{R}=0.05$ and jumps at $x=-50$ and $x=-40$. The characteristic ridge at height $h_{K}$ is not yet formed as the structures collide with the forcing (this is the reason why the DWA was not used for this particular simulation). The fluid that gathers behind the forcing is not enough to form the upstream ridge at $h_{A}$ and the corresponding shock, therefore not inducing type II (Fig. 20, solid line). However, the solution from the jump at $x=-50$ does have
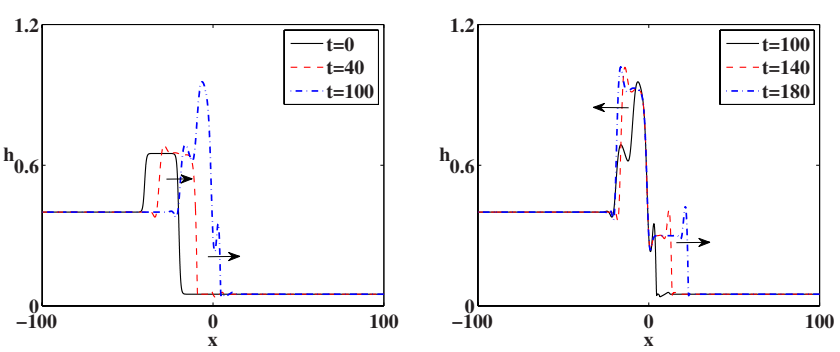

FIG. 22. (Color online) Evolution of the fluid profile for $M$ $=1.5, h_{L}=0.4$, and $h_{R}=0.05$. The fluid in the DWA is absorbed into the fluid gathered behind the forcing, yielding a type II solution with a downstream compressive shock. The downstream behavior is not affected by the DWA because the forcing constrains the flow.

enough mass to form the stable upstream shock. The temporary reservoir of fluid simply leaks out, leading to a type I solution. The formation of the upstream shock connecting $h_{A}$ to $h_{L}$ is the determining factor in creating the stable induced type II solution; consequently the width of the DWA must be sufficiently large to allow for the reservoir fluid to reach the stable height $h_{A}$. In [6], a time-varying $M$ was used to manipulate the behavior of the system in the bistable region in a similar way, using a large $M$ to produce the fluid reservoir rather than introducing a double wave structure to provide this extra mass.

\section{E. Type II solution from a DWA and strong forcing}

If $h_{L}>h_{C}$, then the fluid flow is always constrained by the forcing even after the double wave structure is absorbed, which leads to shocks offset by the mass of fluid in the double wave structure, as shown in Fig. 21 with the transients shown in Fig. 22. The position of the shock is offset depending on the width of the initial condition. Since the initial heights are identical, the masses are unequal. At $t$ $=800$, the transients on the right are nearly identical, while the waves on the left move at the same speed (due to the jump conditions) but are offset due to the difference in mass of the initial conditions; the mass transmitted is limited by the flux determined by $M$. Note that the speed of the leftmoving shock is only accurate after the solution has absorbed transient waves and achieved the predicted intermediate heights. This occurs just after the fluid gathered behind the forcing reaches its maximum height (see Fig. 22, at $t$ $=100$; by $t=140$ the shock has formed). The small perturbation visible near the origin before the downstream transient sweeps over it is the $N$ wave described in Sec. II A and Fig. 14 of that section. The left-moving compressive waves are nearly identical despite the changes in the shape of the initial condition.

\section{CONCLUSION}

We have successfully addressed questions posed in [6] about transient structures and the use of localized forcing to control both transient and steady-state wave structures in a Marangoni-driven thin liquid film. The waves that develop from a given initial profile depend on the boundary data 
$\left(h_{L}, h_{R}\right)$, the forcing shape $\theta(x)$, and the forcing parameter $M$.

We have particularly focused on $N$ waves that appear as early-time solutions. Using a constant initial profile we have demonstrated how a variety of forcing functions and initial conditions can create $N$ waves in numerical simulations. We have also explored solutions arising from monotonic initial conditions that nucleate to form a compressiveundercompressive pair known as a double wave structure. Using an approximation to this structure, we demonstrated that the additional buildup of mass before the wave reaches the forcing region affects the steady-state solution when the forcing parameter is in the moderate (bistable) regime. For strong and weak forcing, the steady state is unaffected by the nucleation.

We hope that future physical experiments will be conducted to verify the existence of these waves. The results of this analysis may be used to control the thickness of a coat- ing flow, using careful choices of forcing function $\theta(x)$, boundary data $h_{L}, h_{R}$, and forcing parameter $M$ to create a microfluidic valve in the forcing region. Future experiments could provide insight into potential applications of this theory to biological and industrial coating flows.

\section{ACKNOWLEDGMENTS}

This work was supported by NSF Grants No. DMS0239125 CAREER, No. DMS-0244498 FRG, and No. DMS0601395 RTG. The authors thank Ryan Haskett, Michael Shearer, Andrew Bernoff, and Tom Witelski for helpful conversations. We also thank Tom Witelski of Duke University, Andrea Bertozzi of the Applied Mathematics Group at UCLA, and the Rose Hills Foundation for support of this work.
[1] A. L. Bertozzi, A. Münch, and M. Shearer, Physica D 134, 431 (1999).

[2] A. M. Cazabat, F. Heslot, S. M. Troian, and P. Carles, Nature (London) 346, 824 (1990).

[3] J. Sur, A. L. Bertozzi, and R. P. Behringer, Phys. Rev. Lett. 90, 126105 (2003).

[4] P. G. LeFloch and M. Shearer, Proc. R. Soc. Edinburgh, Sect. A: Math. 134, 961 (2004).

[5] R. Levy and M. Shearer, Physica D 209, 145 (2005).

[6] R. P. Haskett, T. P. Witelski, and J. Sur, Physica D 209, 117 (2005).

[7] N. Garnier, R. O. Grigoriev, and M. F. Schatz, Phys. Rev. Lett. 91, 054501 (2003).

[8] A. C. Tam, H. K. Park, and C. P. Grigoropoulos, Appl. Surf. Sci. 127-129, 721 (1998).

[9] S. B. G. M. O’Brien, J. Fluid Mech. 254, 649 (1993).

[10] K. Wolke, B. Eitel, M. Schenkl, S. Rmmelin, and R. Schild, Solid State Technol. 39, 87 (1996).

[11] G. B. Whitham, Linear and Nonlinear Waves (Wiley, New York, 1974).
[12] A. L. Bertozzi, A. Münch, X. Fanton, and A. M. Cazabat, Phys. Rev. Lett. 81, 5169 (1998).

[13] A. L. Bertozzi, Not. Am. Math. Soc. 45, 689 (1998).

[14] A. L. Bertozzi and M. Shearer, SIAM J. Math. Anal. 32, 194 (2000).

[15] V. Manukian and S. Schecter, Nonlinearity 22, 85 (2009an y).

[16] A. L. Bertozzi and M. P. Brenner, Phys. Fluids 9, 530 (1997).

[17] A. B. M. Bowen, J. Sur, and R. P. Behringer, Physica D 209, 36 (2005).

[18] J. Sur, T. P. Witelski, and R. P. Behringer, Phys. Rev. Lett. 93, 247803 (2004).

[19] A. Münch, SIAM J. Appl. Math. 62, 2045 (2002).

[20] W. Press, B. Flannery, S. Teukolsky, and W. Vetterling, Numerical Recipes: The Art of Scientific Computing (Cambridge University Press, Cambridge, England, 2007).

[21] P. L. Sachdev and C. S. Rao, Appl. Math. Lett. 13, 1 (2000).

[22] S. Gurbatov, J. Acoust. Soc. Am. 105, 957 (1999).

[23] D. Finkel, C. Kuster, M. Lasater, R. Levy, J. Reese, and I. Ipsen, SIAM Rev. 48, 359 (2006). 